\title{
História da enfermagem obstétrica na Escola de Enfermagem Carlos Chagas: análise sob a perspectiva freidsoniana
}

\author{
History of obstetric nursing at the Nursing School Carlos Chagas: \\ an analysis based on the Freidsonian approach \\ Historia de la enfermería obstétrica en la Escuela de Enfermería Carlos \\ Chagas: análisis bajo la perspectiva de Elliot Freidson
}

Como citar este artigo:

Schreck RSC, Frugoli AG, Santos BM, Carregal FAS, Silva KL, Santos FBO. History of obstetric nursing at the Nursing School Carlos Chagas: an analysis based on the Freidsonian approach. Rev Esc Enferm USP. 2021;55:e03762. https://doi.org/10.1590/S1980-220X2020014703762

Rafaela Siqueira Costa Schreck ${ }^{1}$

Alice Gomes Frugoli ${ }^{2}$

Biannka Melo dos Santos ${ }^{2}$

Fernanda Alves dos Santos Carregal ${ }^{1}$

Kênia Lara da Silva ${ }^{1}$

Fernanda Batista Oliveira Santos ${ }^{2}$

${ }^{1}$ Universidade Federal de Minas Gerais, Escola de Enfermagem, Programa de Pós-graduação em Enfermagem, Belo Horizonte, MG, Brasil.

${ }^{2}$ Universidade Federal de Minas Gerais,

Escola de Enfermagem, Graduação em

Enfermagem, Belo Horizonte, MG, Brasil.
Autor correspondente:

Rafaela Siqueira Costa Schreck

Universidade Federal de Minas Gerais, Escola de Enfermagem

Av. Alfredo Balena, 190, Sala 218 - Campus

Saúde - Bairro Santa Efigênia

CEP 30130-100 - Belo Horizonte, MG, Brasil

rafaelasiqcosta@yahoo.com.br

\section{ABSTRACT}

Objective: to analyze the offering of the first graduate certificate program in nursing in the state of Minas Gerais, called "Graduate Certificate Course in Obstetrics" (Curso de Pós-Graduação em Obstetrícia), by the Nursing School Carlos Chagas, in 1966, and its contribution to the professionalization of obstetric nursing. Method: this is a documentary, socio-historical study, based on the Sociology of Professions by Freidson. Results: the data collected allowed the following thematic composition: academic organization of the graduate certificate program and differentials and discontinuities of the first graduate certificate program at the Nursing School Carlos Chagas, with two categories delineated: "Construction of specific knowledge for obstetric nursing" and "Enhancement of specialized knowledge for scientific notoriety". Conclusion: The first offering of a graduate certificate program in nursing by the Nursing School Carlos Chagas at the Universidade Federal de Minas Gerais was a milestone in the history of nursing in Minas Gerais and of the institution itself for being groundbreaking and having attracted students from other states, with repercussions in Brazil. This protagonism supported the professionalization course in the state and in the country, contributing to the scientific notoriety of the institution and of the professional category.

\section{DESCRIPTORS}

History of Nursing; Obstetric Nursing; Education, Nursing, Graduate; Schools, Nursing. 


\section{INTRODUÇÃO}

A enfermagem brasileira, ao longo da história, vem passando por mudanças significativas na formação educacional. A profissionalização segue a trajetória de organização em torno do modelo de enfermagem anglo-americano, desde os períodos de vinculação às Faculdades de Medicina até o alcance da autonomia no processo formativo e na administração institucional ${ }^{(1-2)}$.

Desde a década de 1920, com as primeiras enfermeiras diplomadas sob o modelo anglo-americano, identificava-se o desejo de consolidação da enfermagem como ciência em torno da construção de conhecimento próprio para a área. Para isso, neste período, "cerca de dezessete enfermeiras diplomadas pela Escola de Enfermagem Anna Nery realizaram cursos de pós-graduação nos Estados Unidos, com bolsa de estudos da Fundação Rockfeller” ${ }^{(3)}$. Mais tarde, nos anos 1940 e 1950, ampliou-se o número de enfermeiras brasileiras em cursos de pós-graduação no exterior ${ }^{(2-3)}$.

Em 1939, entre as primeiras iniciativas de criação de cursos de pós-graduação em enfermagem no Brasil, começou a ser oferecido, em São Paulo, o Curso de Especialização em Enfermagem e Obstetrícia. Após a promulgação da Lei no 775/1949, que dispunha sobre a ampliação do conhecimento especializado no ensino de enfermagem no país, este curso foi incorporado à Escola de Enfermeiras do Hospital São Paulo, atual Escola Paulista de Enfermagem. Na Escola de Enfermagem Anna Nery os primeiros cursos de pós-graduação lato sensu iniciaram-se em 1947, primeiramente para a formação de professores, com vistas a pós-graduação stricto sensu e, a partir de 1948, foram ofertados cursos com o nome de especialização para as áreas de Obstetrícia e Saúde Pública ${ }^{(4)}$.

Em 1955, a Lei no 2.604 regulamentou o exercício profissional da enfermagem no Brasil, discorrendo sobre as seis categorizações existentes na enfermagem na época: Enfermeiro, Auxiliar de Enfermagem, Obstetriz, Parteira, Parteira Prática, Enfermeiro Prático ou Prático de Enfermagem. Essa lei revogou a primeira norma legal que regulou a enfermagem, abordando as lacunas das atribuições dos profissionais, além da referência à titulação necessária para a profissionalização da enfermagem brasileira ${ }^{(5)}$.

Outras iniciativas para atender a necessidade de qualificação profissional no país ocorreram na década de 1950 no ensino lato sensu em enfermagem nas áreas de Pedagogia e Didática aplicada à Enfermagem e Administração de Unidade de Enfermagem na Escola de Enfermagem Rachel Haddock Lobo, atual Escola de Enfermagem da Universidade Estadual do Rio de Janeiro (UFMG), e na Escola de Enfermagem da Universidade de São Paulo (USP) ${ }^{(6)}$.

Na década de 1960, o contexto político de modernização conservadora definiu marcos importantes para o desenvolvimento da formação em enfermagem no país: a primeira Lei de Diretrizes e Bases (LDB) da Educação de 1961 e o Parecer no 977/1965 da Câmara de Ensino Superior, que estabeleceram normas gerais para organização e funcionamento da pós-graduação no Brasil. Iniciaram-se, assim, cursos de enfermagem de especialização e aperfeiçoamento lato sensu com objetivo técnico-profissional de formar profissionais especializados para atender nas áreas curativas e de gerenciamento dos serviços de saúde ${ }^{(2)}$.
A Universidade Federal de Minas Gerais (UFMG) sempre se mostrou na vanguarda das discussões que apontavam para o progresso da educação brasileira. A instituição vem acompanhando as mudanças e vigências do ensino superior nacional e a Escola de Enfermagem Carlos Chagas (EECC), atual Escola de Enfermagem da UFMG (EEUFMG), a segunda escola de enfermagem do país criada em 1933 aos moldes do modelo anglo-americano, tornou-se pioneira na oferta de cursos de pós-graduação em enfermagem no estado, protagonizando os processos de profissionalização em Minas Gerais ${ }^{(1)}$.

O primeiro curso de especialização ofertado pela EECC foi denominado "Curso de Pós-Graduação em Obstetrícia" em $1966^{(1)}$. Ressalta-se que o estado de Minas Gerais possui tradição na formação de enfermeiros especialistas em obstetrícia e a Escola de Enfermagem da UFMG segue, na atualidade, desempenhando um papel importante na história da enfermagem obstétrica, com ampla trajetória no ensino aliado às práticas científicas na assistência à mulher. Considerando o histórico da EEUFMG na área do cuidado obstétrico, a necessidade de definir um campo de saber próprio para a legitimação da profissão frente à notoriedade científica ${ }^{(7)}$, desvelar os caminhos e (des)caminhos percorridos ao longo da história para consolidação da especialização obstétrica torna-se relevante. Os achados deste estudo poderão contribuir para a compreensão da ascensão da Enfermagem no Brasil nos últimos anos, atrelada ao conhecimento histórico ${ }^{(1)}$.

Portanto, o presente trabalho tem como objetivo analisar a oferta da primeira especialização em enfermagem do Estado de Minas Gerais, denominada "Curso de PósGraduação em Obstetrícia”, pela EECC, em 1966, e sua contribuição para a notoriedade científica da categoria profissional enfermagem obstétrica.

\section{MÉTODO}

\section{Desenho do estudo}

Estudo de natureza sócio histórica com análise documental. Considerou-se a perspectiva social, possibilitando a afirmação de que, em história, todas as abordagens estão inscritas nesta ordem e fazem interface entre $\mathrm{si}^{(8)}$.

\section{Cenário}

O cenário de estudo foi a cidade de Belo Horizonte, capital de Minas Gerais, na década de 1960, local e período em que ocorreu a organização e oferta do primeiro curso de Pós-Graduação em Obstetrícia da Escola de Enfermagem Carlos Chagas, atual Escola de Enfermagem da UFMG.

\section{Critérios de SELEÇão}

O critério para seleção de documentos foi a menção ou discurso livre acerca deste curso de especialização.

\section{Coleta de dados}

A coleta documental foi feita no período de junho a setembro de 2019, a partir de seis documentos do acervo do Centro de Memória da Escola de Enfermagem da UFMG (CEMENF), conforme Quadro 1. 
Quadro 1 - Documentos utilizados como fonte primária, separados por código e descrição - Minas Gerais, Brasil, 2019.

\begin{tabular}{|l|r|}
\hline Código & Descrição do documento \\
\hline D1 & Regimento Interno do Curso de Pós-Graduação em Enfermagem Obstetrícia 1966 \\
\hline D2 & Relação de Notas 1966 - Curso de Pós-Graduação em Obstetrícia \\
\hline D3 & Currículo de pós-graduação em Enfermagem Obstétrica \\
\hline E1/E2/E3 & $\begin{array}{c}\text { Entrevistas: Irmã Cecília Bhering/Aparecida Ferreira Moura/Carmelita Pinto Rabelo, respectivamente (versão utilizada: transcrição dos } \\
\text { áudios originais) }\end{array}$ \\
\hline
\end{tabular}

\section{ANÁlISE E TRATAMENTO DOS DADOS}

Após a leitura e condensação, os achados foram organizados considerando o referencial teórico da Sociologia das profissões segundo Eliot Freidson, uma vez que este se mostra assertivo para análises dos processos de profissionalização e construção de saberes especializados. Assim, foram delineadas duas categorias analíticas: "Construção de conhecimento próprio para a enfermagem obstétrica" e "Fortalecimento do saber especializado para notoriedade científica".

\section{Aspectos ÉtIICOS}

O estudo abordou fontes documentais de acervo público, sendo isento de parecer de um Comitê de Ética em Pesquisa e não havendo implicações éticas na citação das personalidades históricas.

\section{RESULTADOS}

\section{Organização aCadêMICa do CURSo de ESPECIALIZAÇÃO}

O curso de Pós-Graduação em Obstetrícia foi dividido em dois períodos de 5 meses: $1^{\circ}$ período: 11 de abril de 1966 a 30 de setembro de 1966 e $2^{\circ}$ período: $1^{\circ}$ de outubro de 1966 a 31 de março de 1967. O curso com duração de, no mínimo, 180 dias de "trabalho escolar efetivo" e 6 horas de "atividades escolares diárias", compreendia ensino teórico-prático, estágios em serviços hospitalares, maternidades em domicílio, ambulatórios e unidades sanitárias. O Quadro 2 apresenta a descrição das atividades curriculares desenvolvidas pelas alunas.

Quadro 2 - Calendário de atividades do Curso de Especialização em Obstetrícia da EECC (1966) - Minas Gerais, Brasil, 2019.

\begin{tabular}{|l|c|}
\hline Período do ano & Atividade \\
\hline 11 de abril de 1966 a 05 de julho de 1966 & Teoria e estágio \\
\hline 06 de julho de 1966 a 20 de julho 1966 & Férias \\
\hline 21 de julho 1966 & Início da contagem de casos atendidos pelas alunas \\
\hline Outubro de 1966 & Entrega do relatório \\
\hline Janeiro de 1967 & Exame final \\
\hline Fevereiro de 1967 & Fim do curso \\
\hline 03 de março 1967 & \\
\hline
\end{tabular}

As professoras do curso eram quatro enfermeiras: Irmã Cecília Bhering, Maria Vitória da Silva, Elza Lanza e, mais ao fim do curso, em 1967, Aparecida Ferreira. As quatro enfermeiras especializaram-se no mesmo local, Escola Paulista de Enfermagem/Hospital São Paulo, em anos próximos, e eram responsáveis pelo conteúdo teórico e prático do curso de pós-graduação em obstetrícia da EECC. Para criação e implementação da especialização da EECC foi necessária uma interlocução com os professores da disciplina de obstetrícia da Faculdade de Medicina da UFMG, que conheciam o renomado trabalho da Irmã Cecília Bhering e apoiaram fortemente a iniciativa. $\mathrm{O}$ segmento básico era ministrado por professores médicos, de acordo com o direcionamento das professoras enfermeiras. O conteúdo era fracionado em três subdivisões, conforme apresentado no Quadro 3.

Quadro 3 - Disciplinas ministradas no Curso de Especialização em Obstetrícia da EECC (1966) - Minas Gerais, Brasil, 2019.

\begin{tabular}{|l|c|}
\hline \multirow{4}{*}{ Disciplinas da Enfermagem } & Enfermagem em Obstetrícia e Ginecologia \\
\cline { 2 - 3 } & Enfermagem em Saúde Pública integrada à Enfermagem Obstétrica \\
\cline { 2 - 2 } & Aulas de Enfermagem Psiquiátrica integrada à Enfermagem Obstétrica \\
\hline \multirow{3}{*}{ Matérias Complementares } & Anatomia e Fisiologia dos Órgãos Genitais \\
\cline { 2 - 3 } & Histologia (embriologia) \\
\cline { 2 - 3 } & Hematologia em Laboratório \\
\hline
\end{tabular}


...continuação

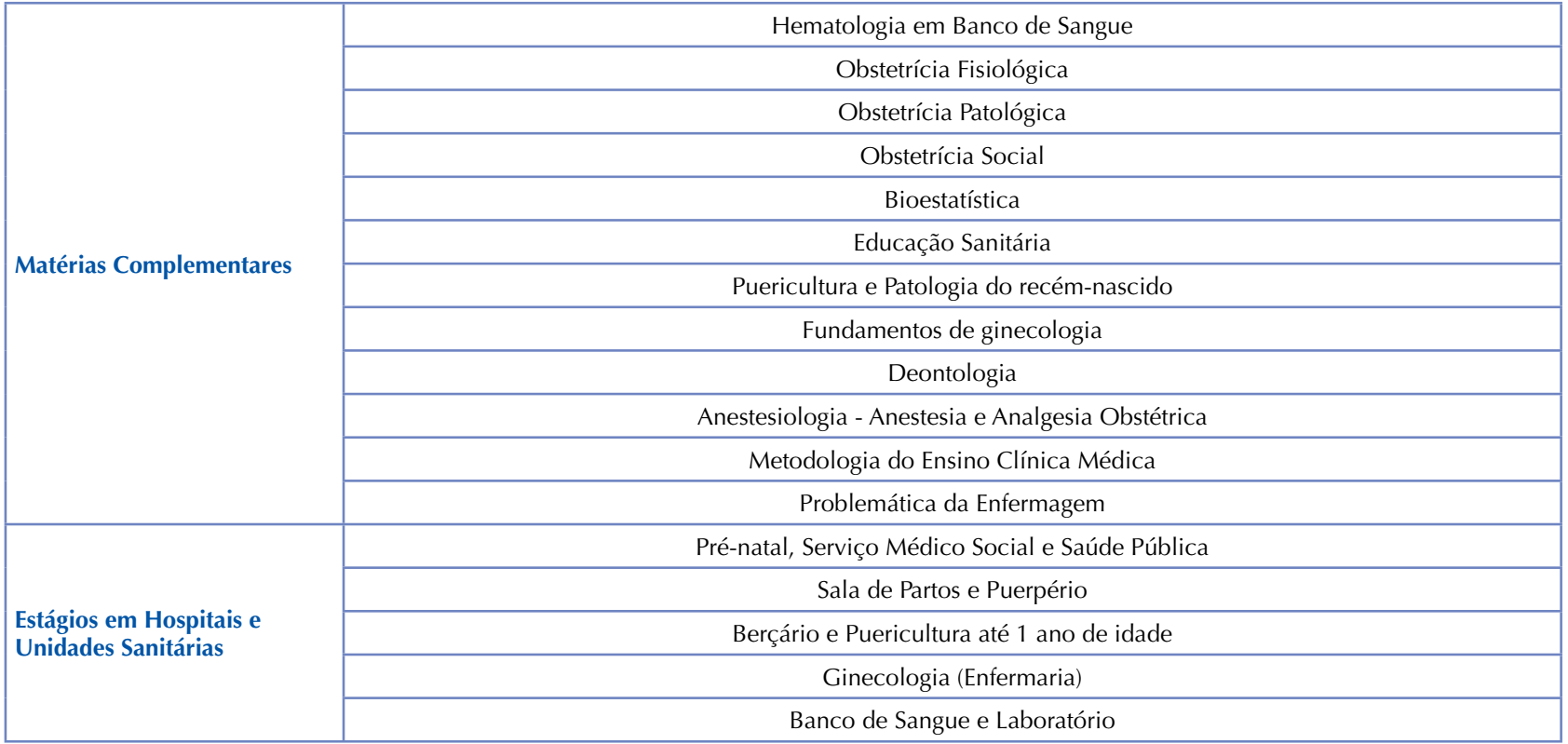

As alunas assistiam às gestantes em continuidade no pré-natal, parto e até 30 dias no pós-parto. Todas as atividades eram supervisionadas pelas professoras, e os casos clínicos acompanhados deveriam ser descritos em relatórios. A exigência era de um total de 270 casos por aluna, divididos entre atendimentos descritos nos documentos, como acompanhamento de gestantes, anamnese pré-natal, partos normais assistidos, avaliação de recém-nascidos normais e patológicos, pré e pós-operatório de cirurgias ginecológicas, puerpérios normais e patológicos e casos sociais.

Os estágios parcial e final davam-se em três etapas, com assistência das especializandas às mulheres em todo o ciclo gravídico-puerperal e aos recém-nascidos. A avaliação das alunas era feita entre as médias das notas do estágio parcial e final, chamada de "Média de estágio" e considerada junto às outras competências exigidas para aprovação final das alunas. Os estágios, denominados internatos, foram todos desenvolvidos em Belo Horizonte, no Hospital São Vicente, atual Hospital das Clínicas da UFMG, Hospital Militar e Santa Casa de Misericórdia. Em geral, a relação entre as enfermeiras e os campos de estágio era respeitosa, com confiança dos médicos e autonomia das enfermeiras nos locais de prática.

Existia uma confiança muito grande dos médicos conosco (...) eles não faziam nada sem vir nos perguntar, nada, eles não receitavam nada sem vir nos perguntar (...) (E1).

Num hospital escola, medicina, nós somos (éramos) responsáveis pelo pré-natal, nós tínhamos um atendimento de 45 mulheres pela manbã e nós passávamos para o médico três a quatro casos (...) e existia participação da mulher (E1).

O curso de especialização em obstetrícia oferecido na EECC seguiu o modelo da Escola Paulista de Medicina, criado por Madre Domineuc, no final da década de 1930. Este modelo foi importante para delinear a organização das disciplinas do curso, pois além de disciplinas relativas ao cuidado biológico, havia enfoque à chamada "Obstetrícia Social". As professoras, juntamente com as alunas, sem apoio ou financiamento da faculdade de medicina, arrecadavam fundos para arcar com os custos do aluguel de uma casa, que recebeu o nome de "Colmeia". Relata-se que pediam dinheiro na porta de igrejas e no cemitério no dia de finados. A casa, localizada na região do Bairro Santa Efigênia, próximo à EECC, tinha como objetivo acolher mulheres em condições precárias e ajustá-las à maternidade.

$\mathrm{Na}$ Colmeia, além das discentes e docentes, residiam as mães e gestantes solteiras, que custeavam a estadia com cuidados das roupas de alunas e professoras. Ao mesmo tempo que moravam com essas mulheres, as alunas desenvolviam trabalhos, evidenciando traços de educação permanente em saúde, muito marcante da práxis da enfermagem.

Nós tínhamos aquela fllosofia (...) toda enfermeira obstétrica tem que dar amparo pra mãe não doar o fliho (...) ela (a mãe) só doa se tem pressão da familia ou se ela não tem para onde ir (E2).

(...) Essa casa foi uma necessidade sentida, nós temos as mãezinhas na maternidade que não (...) tinham seus maridos, que não se responsabilizaram pelo filho (...). Isto é obstetrícia social, resolver o problema, não é deu à luz e tchau tchau (...) (E1).

A casa foi crescendo com o passar dos anos até ser transferida para um novo endereço, onde os trabalhos sociais continuaram independentemente à EEUFMG. A presença da Irmã Cecília Bhering na direção do curso também conferiu um caráter assistencial, acompanhando um processo histórico de formação da enfermagem e de outras profissões da área da saúde com forte influência das necessidades sociais, culturais e políticas da população. A alta qualidade do ensino da EECC garantia às alunas uma formação intelectual e profissional exigente e completa, principalmente em relação ao cuidado integral à mulher e recém-nascido. 
DifERENCIAIS E DESCONTINUIDADES DA PRIMEIRA ESPECIALIZAÇÃO DA EECC

A pós-graduação ofertada pela EECC ficou rapidamente conhecida nacionalmente, atraindo alunas de toda parte do país, como relatado em todas as entrevistas consultadas. A primeira turma era composta por 12 alunas, vindas de várias localidades, dentre elas algumas citadas nas entrevistas (Ceará, Bahia e São Paulo), que receberam o título de enfermeiras obstétricas em uma colação de grau. A expectativa era de que houvesse uma segunda turma para o curso que começou, mas não teve continuidade.

O término da oferta não foi bem explicado na documentação consultada. No entanto, sustentam-se duas hipóteses, ambas relacionadas à mudança de chefia da EECC e do Hospital São Vicente. Uma das hipóteses, defendida por Irmã Cecília Bhering, atribui o fim do curso de pós-graduação à mudança de direção da EECC, apontando Irmã Emília Clarízia, diretora neste período, como uma das opositoras. Segundo Bhering, a nova direção se opôs ao curso sem motivo claro. Por sua vez, Aparecida Ferreira relaciona o término do curso à mudança de gestão do Hospital São Vicente. Ela descreve a sucessão do cargo como "autoritária e sem educação” (E2), às vezes impedindo as enfermeiras de assistirem aos partos, mesmo com toda a documentação e regulamentação do curso apresentada em resposta por elas. Por fim, um contato com um servidor do Hospital Militar organizou a progressão dos estágios da segunda turma para esse hospital.

Sabe-se que a mudança de direção da EECC coincide com o deslocamento de poder entre as religiosas e as leigas. Seria a oposição ao curso uma divergência de interesses entre esses dois grupos? Ou a saída das religiosas da diretoria culminou na perda do principal campo de estágio, consequente com o encerramento do curso? Em ambos os casos, não fica claro na documentação o momento exato em que o curso foi interrompido, deixando em aberto o real motivo do término da especialização.

De toda maneira, o curso da EECC resultou na formação de 12 enfermeiras especialistas em obstetrícia e no pioneirismo dessa instituição na oferta de pós-graduação em Minas Gerais.

Esse curso de especialização merece destaque para ser objeto de um estudo histórico, pois possuía características importantes (formação de um saber próprio; conhecimentos práticos e teóricos; assistência ampliada para a saúde da mulher) na história do desenvolvimento da enfermagem obstétrica, apoiando a profissionalização no estado e no país, ao iniciar um percurso de reconhecimento social da enfermagem especializada no cuidado à mulher e ao binômio mãe e filho.

\section{DISCUSSÃO}

\section{CONSTRUÇÃo DE CONHECIMENTO PRÓPRIO PARA A ENFERMAGEM OBSTÉTRICA}

$\mathrm{Na}$ perspectiva da sociologia das profissões, a profissão é caracterizada como um tipo de ocupação que domina um conhecimento especializado, fundamentado nas credenciais obtidas nas universidades. Desse modo, os delineamentos da profissionalização da enfermagem obstétrica apontam as influências do papel de instituições de ensino na construção do conhecimento próprio e a consequente conquista da reserva de mercado $^{(9)}$.

No Brasil, entre 1922 e 1925, a titulação de especialista em obstetrícia "para as enfermeiras foi atribuída às formandas do Curso de Obstetrícia da Faculdade de Medicina e Cirurgia do Pará"(10). Em 1931, os cursos de partos foram anexados à cadeira de clínica obstétrica das Faculdades de Medicina ${ }^{(11)}$. A regulamentação do ensino de enfermagem no Brasil ocorreu em meados do século XIX, e desde esse período a "profissão sofreu inúmeras modificações e ganhou muito espaço e respeito no âmbito da saúde, principalmente no que concerne à saúde da mulher"(10).

A evolução da sociedade contemporânea está atrelada ao desenvolvimento do conhecimento institucionalizado, forma pela qual se organiza a ocupação dos profissionais no mercado de trabalho. Além disso, há a importância do Estado na regulamentação e controle do exercício profissional ${ }^{(9)}$.

Apesar da formação de apenas uma única turma, a especialização em enfermagem obstétrica da EECC teve grande visibilidade nacional e importância para a trajetória profissional dessa categoria ${ }^{(12)}$, o que pode ser evidenciado pela presença de alunas de outros estados que vieram se especializar em Minas Gerais e pelo modelo de formação voltado para o compromisso social de melhoria das condições de saúde da mulher e do recém-nascido, o que caracteriza ainda hoje a premissa dos valores na formação profissional sustentados pela EEUFMG.

O curso de especialização ofertado pela instituição mineira funcionava de forma autônoma da Faculdade de Medicina, mesmo com a colaboração de professores médicos na estrutura curricular. Por sua vez, o Curso de Enfermagem Obstétrica ofertado, neste mesmo período, pela Escola de Parteiras de São Paulo, era fortemente subordinado ao saber médico e funcionou anexo à Faculdade de Medicina até o início da década de 1970, quando foi incorporado à Escola de Enfermagem da USP(11).

As professoras enfermeiras da EECC, que realizavam também a supervisão dos estágios, capacitavam as alunas para uma atuação profissional centrada em saberes teóricos e práticos próprios da profissão. Essa característica aponta o motivo para a procura pelo curso por alunas vindas de São Paulo, uma vez que, apesar da participação de parteiras, obstetrizes e enfermeiras no ensino, o curso paulista tinha como princípio capacitar enfermeiras para atuarem como auxiliares da prática médica, no parto em domicílio e no hospital ${ }^{(1,13)}$.

Além disso, ressalta-se a particularidade da EECC na formação de enfermeiras especialistas em obstetrícia com conhecimentos teóricos e vivências práticas acerca das condições sociais de vulnerabilidade da saúde da mulher na região, diferentemente de outras instituições que priorizavam uma formação centrada no atendimento hospitalar e de urgência obstétrica ${ }^{(11,13)}$.

A qualidade do ensino da especialização possibilitou a atuação das alunas na Santa Casa de Misericórdia, uma vez 
que, por conta de uma infração às regras em um estágio de obstetrícia da graduação, a Escola de Enfermagem estava proibida de adentrar as dependências dessa instituição desde $1936^{(12)}$. O registro da Santa Casa de Misericórdia como campo de estágio para a pós-graduação pode significar uma reabertura de portas para a EECC nessa instituição, além de evidenciar, a partir do referencial da Sociologia das Profissões ${ }^{(7)}$, o interesse nas alunas, enquanto enfermeiras formadas, para compor o quadro profissional.

As características da formação na EECC garantiram, posteriormente, às egressas, espaço no mercado de trabalho. Há registros de que três recém-formadas da primeira turma ficaram no Hospital das Clínicas ou deram continuidade ao trabalho como enfermeira na UFMG. A credibilidade do curso ofertado pela EECC, juntamente com possíveis outros interesses, inclusive políticos, garantiram à aluna Corina Bastos Crespo uma bolsa de estudos financiada pelo Governo, com publicação no Diário Oficial da União, para despesas durante a especialização ${ }^{(14)}$.

Destaca-se também a trajetória profissional da egressa Vera Moreira de Oliveira, que atuou como professora da Escola Paulista de Enfermagem, em 1972, demonstrando a qualidade do ensino ofertado pela EECC, com a inserção em um campo de atuação fora do estado ${ }^{(15)}$. Nesse cenário, os acontecimentos da primeira turma de pós-graduação da EECC marcam o início de um processo de autonomia profissional, com a construção de um saber próprio da enfermagem obstétrica, em Minas Gerais, e afirmação "como trabalho do campo da saúde, disciplina do conhecimento científico e como uma profissão orientada para o serviço" ${ }^{(16)}$.

A partir do referencial teórico de que "uma profissão alcança sua autonomia quando consegue ela própria exercer controle sobre normas e desempenhos dos seus membros profissionais"(16), pode-se afirmar que a primeira especialização da EECC contribuiu para a construção do conhecimento científico da enfermagem obstétrica, com um ensino coordenado por docentes enfermeiras, uma estrutura curricular com uma importante carga horária de disciplinas baseadas nas práticas da enfermagem e um processo avaliativo entre os pares da profissão $0^{(7,9)}$.

A formulação do ensino assistencial da enfermagem obstétrica no curso de pós-graduação da EECC seguiu os mesmos princípios do trabalho profissional da Enfermagem, com a organização de um conjunto de ações de saúde e realização de práticas de cuidado, acompanhando também as características de formação de outras instituições no país, como a Escola Anna Nery de Enfermagem, da UFRJ e Escola de Enfermagem do Hospital São Paulo ${ }^{(11,13)}$.

A estrutura curricular do curso, fortemente marcado pela prática, com a exigência de uma maior carga horária de "trabalho escolar efetivo", demonstra a necessidade da formação, em serviço, de um profissional com destreza técnica, associada ao conhecimento teórico e científico para a assistência à mulher e ao recém-nascido. Esse modelo de formação, atualmente, é o recomendado e defendido pelas diretrizes pedagógicas dos programas de residência em enfermagem obstétrica e multiprofissional, que têm como estratégia o ensino teórico-prático em serviço, para possibilitar uma aprendizagem vivenciada do cuidado e da educação em saúde $^{(17)}$. As disciplinas teóricas do curso, juntamente com os estágios nos campos de prática hospitalares, conforme é possível observar na experiência profissional de pelo menos duas enfermeiras especialistas formadas pelo curso, possibilitam a construção de um conhecimento próprio para a enfermagem obstétrica, formando enfermeiras aptas a atuarem com qualidade e segurança nos cuidados às mulheres durante a gestação, parto e puerpério.

O processo de especialização das enfermeiras, dentro do ambiente formal da Universidade, inaugura uma trajetória de rupturas e continuidades na formação de enfermeiras obstétricas, em Minas Gerais, marcada, ainda hoje, por enfrentamentos e disputas por espaços de $\operatorname{atuação~}^{(17-18)}$.

\section{FORTALECIMENTO DO SABER CIENTífICO PARA NOTORIEDADE CIENTÍFICA}

Os cursos de especialização de enfermagem obstétrica, no Brasil, iniciam a construção de um saber especializado e específico para a área, centrado no cuidado à mulher e família, na humanização do parto e nascimento, com redução das intervenções desnecessárias para redução da mortalidade materna e neonatal. A Especialização de 1966 da EECC apresentou elementos curriculares que foram essenciais para o destaque do curso no cenário nacional, inclusive atraindo alunas de outros estados, inaugurando a trajetória dessa instituição na formação de profissionais qualificados.

Por meio da análise da organização curricular do curso da EECC, é possível afirmar que disciplinas obrigatórias de "Enfermagem Psiquiátrica integrada à Enfermagem Obstétrica", "Obstetrícia Social” e estágio em "Serviço Médico-Social" demonstram uma preocupação do corpo docente com a preparação das enfermeiras alunas para atuarem em um panorama mais ampliado da saúde da mulher, abordando particularidades de grupos populacionais, condições sociais e econômicas.

Nos dias atuais, a assistência ao parto, no Brasil, é caracterizada por intensa medicalização do corpo feminino, também relacionada às características socioculturais e econômicas da população, políticas de saúde, iniquidade e exclusão, resultando em um alto índice de cesarianas e mortalidade materna e perinatal, sobrecarregando os sistemas social e financeiro. O Brasil está entre os países com o maior número dessas cirurgias no mundo, sendo que a cada 10 partos realizados em maternidades particulares no Brasil, 8,5 são cesáreas, ultrapassando a recomendação de 1,5 da Organização Mundial de Saúde (OMS) ${ }^{(19-20)}$.

Uma estratégia para redução do número de cesarianas e, consequente, diminuição das taxas de mortalidade materna, foi o investimento do Governo Federal na formação de profissionais de enfermagem obstétrica, uma vez que estes são capacitados para a assistência ao parto normal de risco habitual. Além disso, a enfermagem obstétrica, ao construir um saber específico, conquistou um espaço diferenciado nos serviços de saúde, defendendo um nascimento seguro e livre de intervenções cirúrgicas e medicamentosas desnecessárias ${ }^{(20-21)}$.

Assim, as políticas públicas passaram a priorizar a formação de profissionais da enfermagem obstétrica. Um marco importante foi a implantação, em 1984, do Programa de Assistência 
Integral à Saúde da Mulher (PAISM), do Ministério da Saúde (MS), que tinha como principal objetivo a garantia de uma assistência integral à saúde da mulher e da equidade de gênero. O financiamento de cursos de especialização em enfermagem obstétrica, desde 1999, foi uma estratégia do MS para alcançar estes princípios, em ação conjunta com universidades e secretarias de saúde do país ${ }^{(1,22)}$. Nesta perspectiva, destaca-se a UFMG, devido ao seu papel histórico de ensino e formação dessa categoria profissional, como polo de coordenação e execução dos projetos de ensino e pesquisa na área.

Em 2000, na busca pela melhoria da assistência materna e perinatal, foi lançado também o Programa de Humanização no Pré-natal e Nascimento (PHPN) para, juntamente com a iniciativa de formação da enfermagem obstétrica, garantir uma maior qualidade no atendimento à gestante, parturiente, puérpera e ao recém-nascido ${ }^{(10)}$. No entanto, a inserção da enfermagem obstétrica no país, ainda hoje, enfrenta algumas das mesmas dificuldades do início da sua formação, como os confrontos com a categoria médica, uma vez que grande parte dessa desconsidera a importância do trabalho desempenhado pelo enfermeiro(a) obstetra para uma assistência segura e humanizada ao parto normal ${ }^{(10,23)}$.

Assim, a Escola de Enfermagem da UFMG, pioneira do ensino da profissão no Estado, contribuiu significativamente com a construção do conhecimento próprio de enfermagem, possibilitando a obtenção do conhecimento especializado e as credenciais necessárias para amparar a reserva de mercado de trabalho.

Considera-se que o processo de construção de um saber específico para a enfermagem obstétrica mineira, pautado em um cuidado humanizado e voltado à saúde pública, teve como marco esse curso de especialização. No entanto, este estudo não pode generalizar a trajetória dos cursos de enfermagem obstétrica no país, uma vez que reconhece a influência de contextos socioculturais, políticos e históricos distintos para cada região brasileira que alteraram as trajetórias de profissionalização em cada localidade.

Este trabalho faz-se relevante por possibilitar a compreensão do processo de formação educacional e prática das enfermeiras obstétricas como campo de conhecimento próprio e especializado, buscando o entendimento do progresso e das dificuldades da profissão. Além disso, possibilita a análise acerca da formulação de diretrizes e normativas que contemplam as especificidades do ensino e prática da enfermagem obstétrica.

As lacunas deixadas nos documentos, como o não esclarecimento do motivo e momento de término do curso e informações conflitantes entre as entrevistas, apresentaram-se como limitações do estudo. No entanto, foi possível delinear a oferta de pós-graduação e sugerir hipóteses para seu término, bem como avaliar a sua importância.

\section{CONCLUSÃO}

A primeira oferta de um curso de especialização em enfermagem pela EEUFMG representou um marco na história da enfermagem mineira e da própria instituição, por ser pioneiro, ter atraído quatro estudantes de fora do estado, alcançando uma repercussão considerável, nesse momento, para além de Minas Gerais. O protagonismo da EEUFMG apoiou o curso da profissionalização no estado e no país e contribuiu para a notoriedade científica dessa instituição e da categoria profissional. Destaca-se o conhecimento histórico como uma ferramenta de compreensão do homem enquanto ser que constrói seu espaço e tempo e como instrumento de transformação da sociedade.

\section{RESUMO}

Objetivo: Analisar a oferta da primeira especialização em enfermagem do estado de Minas Gerais, denominada "Curso de PósGraduação em Obstetrícia”, pela Escola de Enfermagem Carlos Chagas, em 1966, e sua contribuição para a profissionalização da enfermagem obstétrica. Método: Estudo sócio-histórico documental, pautado na Sociologia das Profissões segundo Eliot Freidson. Resultados: Os dados coletados possibilitaram a seguinte composição temática: organização acadêmica do curso de especialização e diferenciais e descontinuidades da primeira especialização da Escola de Enfermagem Carlos Chagas, sendo delineadas duas categorias: "Construção de conhecimento próprio para a enfermagem obstétrica" e "Fortalecimento do saber especializado para notoriedade científica". Conclusão: A primeira oferta de um curso de especialização em enfermagem pela Escola de Enfermagem Carlos Chagas da Universidade Federal de Minas Gerais representou um marco na história da enfermagem mineira e da própria instituição por ser pioneira e ter atraído estudantes de fora do estado, com repercussão pelo Brasil. Este protagonismo apoiou o curso da profissionalização no estado e no país, contribuindo para notoriedade científica da instituição e da categoria profissional.

\section{DESCRITORES}

História da Enfermagem; Enfermagem Obstétrica; Educação de Pós-graduação em Enfermagem; Especialização; Escolas de Enfermagem.

\section{RESUMEN}

Objetivo: Analizar la oferta del primer curso de especialización en enfermería de la provincia de Minas Gerais, denominado "Curso de postgrado en Obstetricia”, ofertado por la Escuela de Enfermería Carlos Chagas en 1966 y su contribución para la profesionalización de la enfermería obstétrica. Método: Estudio socio-histórico documental basado en la Sociología de las Profesiones de Elliot Freidson. Resultados: Los datos colectados permitieron la siguiente composición temática: Organización académica del curso de especialización y diferenciales y discontinuidades de la primera especialización de la Escuela de Enfermería Carlos Chagas en dos categorías: "Construcción de conocimiento propio para la enfermería obstétrica" y "Fortalecimiento del saber especializado para notoriedad científica”. Conclusión: La primera oferta de un curso de especialización en enfermería por la Escuela de Enfermería Carlos Chagas de la Universidad Federal de Minas Gerais representó un hito de la enfermería minera y de la propia institución, por ser pionera y haber captado estudiantes de otras provincias con repercusión por el país. Este protagonismo apoyó la trayectoria de la profesionalización en la provincia y en Brasil con contribución científica y profesional.

\section{DESCRIPTORES}

Historia de la Enfermería; Enfermería Obstétrica; Educación de Postgrado en Enfermería; Facultades de Enfermería. 


\section{REFERÊNCIAS}

1. Santos FBO, Carregal FAS, Rodrigues RD, Marques RC. História da enfermagem brasileira (1950-2004): o que tem sido discutido na literatura?. Rev Enferm Cent.-Oeste Min. 2018;8:e1876. https://doi.org/10.19175/recom.v8i0.1876

2. Mattia BJ, Kleba ME, Prado ML. Nursing training and professional practice: an integrative review of literature. Rev Bras Enferm. 2018;71(4):2039-49. https://doi.org/10.1590/0034-7167-2016-0504

3. Santos TCF, Gomes MLB. Nexus between postgraduation and nursing research in Brazil. Rev Bras Enferm. 2007;60(16):91-95. https://doi. org/10.1590/S0034-71672007000100017

4. Carvalho AC. Associação Brasileira de Enfermagem: Documentário, 1926-1976. Brasília, DF: ABEn; 1976.

5. Brasil. Lei n 2.604, de 17 de setembro de 1955. Regula o Exercício da Enfermagem Profissional. Diário Oficial União, Brasília, DF, 1955 Sept 21.

6. Pimentel MRAR, Xavier ML. Faculdade de enfermagem da universidade do estado do Rio de Janeiro: 70 anos de sua trajetória. Hist Enferm Rev Eletrônica [Internet]. 2018 [cited 2020 Sept 29];9(2):86-8. Available from: http://here.abennacional.org.br/here/v9/n2/_EDITORIAL-1_ portugues.pdf

7. Freidson E. Renascimento do profissionalismo: teoria, profecia e política. São Paulo: Edusp; 1998.

8. Padilha MI, Bellaguarda MLR, Nelson S, Maia ARC, Costa R. The use of sources in historical research. Texto Contexto Enferm. 2017;26(4):e2760017. https://doi.org/10.1590/0104-07072017002760017

9. Freidson E. Para uma análise comparada das profissões: a institucionalização do discurso e do conhecimento formais. Rev Bras Cien Soc [Internet]. 1996 [cited 2020 Mar 19];11(31):141-55. Available from: http://www.cff.org.br/userfiles/60\%20-\%20FREIDSON\%20E\%20\%20 Para\%20uma\%20analise\%20comparada\%20das\%20profissoes_1996.pdf

10. Sena CD, Santos TCS, Carvalho CMF, Sá ACM, Paixão GPN. [Progress and setbacks obstetrical nursing in Brazil]. Rev Enferm UFSM. 2012;2(3):523-9. Portuguese. https://doi.org/10.5902/217976923365

11. Freitas GF, Bonini BB, Silva EC, Araújo TA, Mattozinho FCB. [School of Nursing, University of São Paulo: traces the history of the professionalization of nursing in Brazil]. Cult Cuid [Internet]. 2016 [cited 2020 Mar 19];20(46). Portuguese. Available from: https://rua. ua.es/dspace/bitstream/10045/61753/1/CultCuid_46_07.pdf

12. Santos FBO, Marques, RDC. Nurses graduated from Escola de Enfermagem Carlos Chagas: work áreas: 1936-1948. Esc Anna Nery. 2015;19(2):363-8. https://doi.org/10.5935/1414-8145.20150050

13. Cardili CVC, Sanna MC. Events that preceded Paulista Nursing School Federalization. Esc Anna Nery. 2015;19(1):24-32. https://doi. org/10.5935/1414-8145.20150004

14. Minas Gerais. Lei Ordinária No 3.335, de 23 de dezembro de 1964 [cited 2020 Feb 22]. Abre à Secretaria de Estado da Saúde o Crédito Especial de CR\$11.403.721,90. Available from: https://leisestaduais.com.br/mg/lei-ordinaria-n-3335-1964-minas-gerais-abre-a-secretariade-estado-da-saude-o-credito-especial-de-cr-11-403-721-90

15. Cruz AV, Augusto E, Yuta K, Souza MF, Castro RAP, Oliveira VM. Formação dos profissionais de enfermagem dos três níveis estudo dos recursos financeiros das escolas de enfermagem do país. Rev Bras Enferm. 1972;25(4):43-51. https://doi.org/10.1590/0034716719720004000007

16. Bellaguarda MLR, Padilha MI, Pereira Neto AF, Pires D, Peres MAA. [Reflection on the legitimacy of the autonomy at nursing in the field of the health professions in the light of Eliot Freidson's ideas]. Esc Anna Nery. 2013;17(2):369-74. Portuguese. https://doi.org/10.1590/ S1414-81452013000200023

17. Costa RLL, Barbosa CO. [Legal responsibility of the Obstetric Nurse in labor assistance]. Rev Enferm Contemp. 2019;8(2):164-71. Portuguese. https://doi.org/10.17267/2317-3378rec.v8i2.2239

18. Teixeira KRB, Queirós PJP, Pereira LA, Peres MAA, Almeida Filho AJ, Santos TCF. Associative organization of nursing: struggles for the social recognition of the profession (1943-1946). Rev Bras Enferm. 2017;70(5):1132-40. https://doi.org/10.1590/0034-7167-2017-0186

19. Vega CEP, Soares VMN, Nasr AMNF. [Late maternal mortality: comparison of maternal mortality committees in Brazil]. Cad Saúde Pública. 2017;33(3):e00197315. Portuguese. https://doi.org/10.1590/0102-311x00197315

20. World Health Organization. Statement on Caesarean Section Rates [Internet]. Geneva: World Health Organization; 2015 [cited 2020 Mar 8]. Available from: https://apps.who.int/iris/bitstream/handle/10665/161442/WHO_RHR_15.02_eng.pdf?sequence=1.

21. Brasil. Ministério da Saúde. Comissão Nacional de Incorporação de Tecnologias no SUS. Diretriz Nacional de Assistência ao Parto Normal. Relatório de Recomendação [Internet]. Brasília, DF: Ministério da Saude; 2016 [cited 2020 May 2]. Available from: http://conitec.gov.br/ images/Consultas/2016/Relatorio_Diretriz-PartoNormal_CP.pdf

22. Brasil. Ministério da Saúde. Secretaria de Atenção à Saúde. Núcleo Técnico da Política Nacional de Humanização. Política Nacional de Humanização: documento base para gestores e trabalhadores do SUS [Internet]. 3th ed. Brasília. DF: Ministério da Saúde; 2010 [cited 2020 Feb 12]. Available from: http://bvsms.saude.gov.br/bvs/publicacoes/humanizasus_documento_gestores_trabalhadores_sus.pdf

23. Costa RLM, Costa ILS. [A point of resistance: nursing, medicine and gender in the hospital contexto]. Cad Espaço Feminino. 2017;30(2):357-66. Portuguese. https://doi.org/10.14393/CEF-v30n2-2017-20 